\title{
LETTER-TO-THE-EDITOR
}

\section{Staphylococcus aureus Nasal Carriage Among Health Care Workers in a Nepal Hospital}

\author{
Bidya Shrestha $^{1}$, Bharat Mani Pokhrel ${ }^{2}$ and Tribhuban M Mohapatra ${ }^{1}$ \\ ${ }^{1}$ Department of Microbiology, Institute of Medical Sciences, Banaras Hindu University, Varanasi, India; ${ }^{2}$ Department of Microbiology, Institute \\ of Medicine, Tribhuvan University, Kathmandu, Nepal
}

Humans are natural reservoir for Staphylococcus aureus and the common site of colonization for Methicillin resistant Staphylococcus aureus (MRSA) is the anterior nares. MRSA strains may be introduced by colonized hospital personnel and serve as the major reservoir of MRSA [1].

Colonized healthcare workers (HCWs) transfer such strains to patients or they transfer the organisms from one patient to another by their hands leading to epidemics in chronic care facilities [2]. Transient colonization has been documented up to $50 \%$ among the HCWs [3]. However, long term MRSA colonization among HCWs is not frequent $(<5 \%)$ [1]. The carriers of MRSA are at risk of developing endogenous infection [4]. Colonization of anterior nares with MRSA carries a significantly higher risk of infection than colonization by sensitive strains [5].

Present study, the first of its kind in Nepal, was designed to determine the prevalence of $S$. aureus and MRSA carriage among HCWs.

Nasal swabs ( $\mathrm{n}=258$ ) from both the anterior nares of 129HCWs working in a tertiary care teaching hospital was collected with consent during August to Sept 2008. Of 129 participants from 17 wards, 23 were doctors, 63 were nursing staff, 20 were nursing students and 23 were ward attendants. Of them 111 were female and 18 were male. Their mean age was 32.29 years and age range was 19 to 61 years. The isolation and identification of $S$. aureus was done by following ASM 2004 [6]. Antibiotic sensitivity of $S$. aureus and identification of MRSA and other tests were done by following CLSI 2007 [7]. Reference strains S. aureus ATCC 25923 and ATCC 43300 were used as negative and positive control respectively. Chi square test was used for the statistical analysis.

Of 129 HCWs, $27.13 \%(n=35)$ were identified as nasal carriers of S. aureus. MRSA was isolated from $2.32 \%(\mathrm{n}=3)$ of participants which were heterogeneous MRSA identified on the basis of oxacillin and cefoxitin (Oxoid) by disc diffusion test, oxacillin screen agar test, oxacillin minimum inhibitory concentration by E test (AB biodisk) and MRSA screen test (Denka Seiken, Japan). Of these 3 MRSA isolates, 2 also exhibited erythromycin inducible clindamycin resistance. All isolates were sensitive to vancomycin and teicoplanin. Most isolates except MRSA were sensitive to most of the FDA recommended antibiotics [7]. One MRSA isolate was negative for $\beta$ lactamase

Received on 19 April 2009; revised 11 August 2009.

Address for correspondence: Dr. Bidya Shrestha.Department of Microbiology. Institute of Medical Sciences, Banaras Hindu University, Varanasi, India. E-mail: B_shrestha_07@hotmail.com.

The Brazilian Journal of Infectious Diseases

2009;13(5):322.

(C) 2009 by The Brazilian Journal of Infectious Diseases and Contexto Publishing. All rights reserved. test by chromogenic (nitrocefin disc, BBL), iodometric and acidimetric methods. MRSA were isolated from a doctor, a nursing staff and a nursing student. Nasal carriage was least 21.73\% among doctors and was highest $43.47 \%$ among ward attendants ( $>0.05$ ) and was 30\% among nursing students and 22.22\% among nursing staff. Among doctors, the carriage was higher $28.57 \%$ among males compared to females $11.11 \%$. Carriage was high $47.3 \%$ among female attendants as compared to (25\%) among male attendants $(\mathrm{p}>0.05)$. The increasing trend of nasal carriage was observed with increase in age ( $>0.05)$. More than $50 \%$ carriage rate was found in HCWs serving in post operative wards. Probably due to the antimicrobial selection pressure MRSA spreads more easily than methicillin sensitive $S$. aureus (MSSA). Nasal MRSA colonization carries a significantly greater risk of infection than MSSA [5] and increases the risk of infection by 4 fold [8]. Therefore, for the sake of the HCWs themselves and for limiting staphylococcal nosocomial infections, S. aureus nasal carrier healthcare workers should be identified and trained to practice infection control measures. HCWs who acquire MRSA in hospital transmit the organism to their household eventually spreading such nosocomially acquired multidrug resistant bacteria in the community. Therefore, such carriage study should be conducted at a regular basis in all health sectors followed by the treatment of the identified carriers.

\section{References}

1. Klimek J. J., Marsik F. J., Bartlett R. C., et al. Clinical epidemiology and bacteriologic observations of an outbreak of methicillin resistant Staphylococcus aureus at a large community hospital. Am J Med. 1976;61:340-5.

2. Harbarth S., Liassine N., Dhaan S., et al. Risk factors for persistent carriage of methicillin resistant Staphylococcus aureus. Clin Infect Dis. 2001; 31: 1380-1385.

3. Cookson B., Peters B., Webster M., et al. Staff carriage of epidemic methicillin resistant Staphylococcus aureus. J Clin Microbiol. 1989. 27(7): 1471-1476.

4. Dar J. A., Thokar M. A., Khan J. A., et al. Molecular epidemiology of clinical and carrier strains of methicillin resistant Staphylococcus aureus (MRSA) in the hospital settings of north India. Ann Clin Microbiol Antimicrob. 2006; 5: 22

5. Ansesio A., Guerrero A., Quereda C., et al. Colonization and infection with methicillin resistant Staphylococcus aureus: associated factors and eradication. Infect contol Hosp Epidemiol. 1996; 17: 20-28.

6. Isenberg H D., Clinical microbiology procedure handbook Vol 2, American Society for Microbiology. 2004. 5.5.1-5.6.3

7. Clinical Laboratory Standards Institute. Performance standard for antimicrobial susceptibility testing: seventeenth informational supplement M100- S17. Clinical Laboratory Standards Institute, Wayne, PA, USA. 2007.

8. Safdar N., Bradley E. A. The risk of infection after nasal colonization with Staphylococcus aureus. Am J Med. 2008;121(4): 310-315. 